\title{
Homocysteine and the Risk of Cardiovascular
}

\section{Events and All-Cause Death in Elderly Population: A Community-Based Prospective Cohort Study}

This article was published in the following Dove Press journal:

Therapeutics and Clinical Risk Management

\author{
Zhongying Zhang ${ }^{\prime}{ }^{\prime}$ \\ Xiang $\mathrm{Gu}^{2}$ \\ Xianghua Fang ${ }^{3}$ \\ Zhe Tang ${ }^{4}$ \\ Shaochen Guan $\mathbb{D}^{3}$ \\ Hongjun Liu $^{3}$ \\ Xiaoguang $\mathrm{Wu}^{3}$ \\ Chunxiu Wang $\mathbb{D}^{3}$ \\ Yan Zhao ${ }^{5}$
}

'Geriatric Department, Evidence-Based Medical Center, Xuanwu Hospital, Capital Medical University, Beijing, People's Republic of China; ${ }^{2}$ Medical Affair Department, Beijing Friendship Hospital, Capital Medical University, Beijing, People's Republic of China;

${ }^{3}$ Evidence-Based Medical Center, Xuanwu Hospital, Capital Medical University, Beijing, People's Republic of China; ${ }^{4}$ Beijing Geriatric Healthcare Center, Xuanwu Hospital, Capital Medical University, Beijing, People's Republic of China; ${ }^{5}$ Education Department, Xuanwu Hospital, Capital Medical University, Beijing, People's Republic of China
Correspondence: Xianghua Fang Evidence-Based Medical Center, Xuanwu Hospital, Capital Medical University, No. 45 Changchun Street, Beijing 100053 ,

People's Republic of China

$\mathrm{Tel} / \mathrm{Fax}+86-010-63153439$

Email fangxh_2015@126.com
Background: The association between homocysteine and cardiovascular diseases (CVD) and all-cause death was inconclusive. A community-based prospective cohort study was carried out in Beijing to evaluate this association in elderly population for more effective clinical prediction and primary prevention of CVD.

Patients and Methods: Participants were randomly selected from Beijing, China. Questionnaire survey, physical examinations, and laboratory tests were carried out to collect baseline information and investigate clinical characteristics. Each participant was predetermined to be followed by 5 years. CVD events and death were collected as primary variables. A Cox regression analysis was performed to assess the risk of CVD events, CVD death, and all-cause death contributed by homocysteine as well as some other risk factors.

Results: A total of 1257 participants with an average age of 69.16 years were enrolled in this study. After adjusting for confounders, the hazard ratios (HRs) and $95 \%$ confidence intervals of CVD event, CVD death, and all-cause death caused by intermediate-to-severe hyperhomocysteinemia as compared with normal homocysteine levels were 1.68 (95\% CI 1.06-2.67), 1.97 (95\% CI $0.95-4.29)$ and 2.02 (95\% CI 1.26-3.24), respectively. Intermediate-to-severe hyperhomocysteinemia increased the risks of CVD event (HR 2.07, 95\% CI 1.01-4.26) and all-cause death (HR 3.08, 95\% CI 1.56-6.07) among male participants. However, the positive association was not statistically significant among female participants (HR 1.59, 95\% CI 0.83-3.04 for CVD event and HR $0.90,95 \%$ CI $0.52-6.07$ for all-cause death). Every $5 \mu \mathrm{mol} / \mathrm{L}$ increment in homocysteine concentration was shown to be associated with a 4\% (HR 1.04, 95\% CI 1.01-1.07) and 5\% (HR 1.05, 95\% CI 1.01-1.07) higher risk of CVD events and all-cause death in all participants. There was no significant association between moderate hyperhomocysteinemia and the risk of the CVD events and all-cause death.

Conclusion: Intermediate-to-severe hyperhomocysteinemia was significantly associated with CVD events and all-cause death in elderly population without a history of ischemia or congestive heart failure (CHF). The positive association was pronounced among males.

Keywords: homocysteine, cardiovascular disease, all-cause death, prospective cohort study

\section{Introduction}

Cardiovascular disease (CVD) is now recognized as the leading cause of death in the world. ${ }^{1}$ In China, stroke has become a predominant disease and is the leading cause of permanent disability. ${ }^{2}$ Coronary heart disease also confers a huge health burden. Conventional risk factors such as hypertension, dyslipidemia, diabetes mellitus and smoking do not fully explain the further CVD events and death. ${ }^{3,4}$ 
Some epidemiological studies demonstrated that homocysteine is an independent risk factor for $\mathrm{CVD}^{5-7}$ and is prone to stroke. ${ }^{8,9}$ However, homocysteine-lowering therapy trials were not beneficial for secondary prevention of CVD. ${ }^{10}$ Recently, a large homocysteine-lowering trial among hypertensives in China without a history of stroke or myocardial infarction led to a $21 \%$ reduction in the risk of stroke, which suggested that homocysteine may be associated with primary prevention of CVD. ${ }^{11}$ Additionally, homocysteine is possibly a graded risk factor for CVD, ${ }^{12,13}$ thus moderate hyperhomocysteinemia may not be associated with further CVD events.

A post hoc analysis of the Vitamin Intervention for Stroke Prevention (VISP) trial demonstrated that homocysteinelowering therapy was associated with reduced risk of stroke, myocardial infarction, or death by $24 \%$ among individuals older than 67 , whereas the benefit effect did not exist among individuals younger than $67 .{ }^{14}$ Therefore, the association between homocysteine and CVD appears to be more pronounced in the elderly.

Due to the rapid changing of lifestyle and the cultural diversity in different parts of China, it is necessary to reexamine the impact of homocysteine on the health of elderly. Considering that the association between homocysteine and CVD remains controversial at present, we thus hypothesized that elevated homocysteine was independently associated with CVD events and all-cause death in the elderly Chinese population, which has important clinical and public health implications. Following the Beijing Longitudinal Study of Aging (BLSA) initiated in 1992, a community-based prospective cohort study was carried out in Beijing as an extension of BLSA to test the aforementioned hypothesis from 2009.

\section{Patients and Methods Study Design and Population}

BLSA aimed to investigate the health status of elder population in Beijing, the capital city and the most important city in northern China. The study design, implementation, and part of the results of BLSA have been reported previously. ${ }^{15-17}$ In this study, the selection of study population was carried out by a stratification-random-clustering procedure to ensure the representativeness of the population aged 55 and above in Beijing. Briefly, the participants were randomly selected from 3 out of 18 administrative districts, including 8 urban districts, 5 suburban districts, and 5 country districts of Beijing. All the residents aged 55 and above from specific communities or villages in the selected districts were invited to join the baseline interview and questionnaire survey. The inclusion criteria were (1) living in the selected district by randomized sampling procedure, (2) can participate in normal communication, (3) can read and fill out the questionnaire, (4) has signed the informed consent. Exclusion criteria were (1) with history of myocardial infarction, (2) with history of ischemia, (3) with congestive heart failure (CHF), (4) with history of stroke, (5) refused to have his/ her blood sampled, (6) refused to sign informed consent.

The Ethics Committee of Xuanwu Hospital, Capital Medical University approved this study. This study was conducted in accordance with the Declaration of Helsinki. All participants signed an informed consent form.

\section{Baseline Information}

From June to August 2009, face to face interview and questionnaire survey were carried out to collect the baseline information from each participant by well-trained investigators. Essentially, the questionnaire included the information of demographics, medical history, lifestyle, diet habit, consumption of alcohol and cigarettes, and the use of medications. All participants were required to answer all the questions to the best of their knowledge.

Physical examinations were carried out at designated hospitals or on-site by well-trained investigators, including measuring blood pressure, pulse pressure, body mass index, etc. Blood pressure was measured according to a standard protocol. After a 5 minutes' rest, sitting blood pressure was measured with a digital blood pressure monitor (Omron HEM-4021, Omron, Kyoto, Japan). The measurements were duplicated for each participant on the non-dominant arm with a 2-minute interval. The mean value was used for analysis. Pulse pressure was calculated as systolic minus diastolic pressure. Hypertension was diagnosed as a systolic blood pressure $\geq 140 \mathrm{mmHg}$ and/or a diastolic blood pressure $\geq 90 \mathrm{mmHg}$ or medical history, or currently using of antihypertensive medications, according to the Joint National Committee guideline (JNC VII). ${ }^{18}$

\section{Laboratory Examinations}

Serum total homocysteine concentration was measured by enzymatic cycling assay with Abbott ARCHITECT ${ }^{\circledR}$ System (Architect-i 2000; Abbott, TX, USA) with a minimal detectable concentration of $<0.5 \mu \mathrm{mol} / \mathrm{L}$ and intra-assay and interassay variation coefficients of variation $<7.0 \% .{ }^{19} \mathrm{~A}$ normal level of homocysteine concentration was considered to be $<15 \mu \mathrm{mol} / \mathrm{L}$, whereas hyperhomocysteinemia was defined as 
a concentration of total homocysteine $\geq 15 \mu \mathrm{mol} / \mathrm{L}$. Moderate and intermediate-to-severe hyperhomocysteinemia referred to concentrations equal or more than $15 \mu \mathrm{mol} / \mathrm{L}$ but less than $30 \mu \mathrm{mol} / \mathrm{L}$, and above $30 \mu \mathrm{mol} / 1$, respectively. ${ }^{20}$

Fasting blood samples were collected in the following morning after the completion of questionnaire. The procedures of blood sampling have been reported elsewhere. ${ }^{16}$ In short, all venous blood samples were collected after 12hour fasting and were centrifuged within $1 \mathrm{~h}$ at $3000 \mathrm{rpm}$ for $15 \mathrm{~min}$ right after collection. The separated serum samples were stored in a refrigerator at $2-8^{\circ} \mathrm{C}$ until testing. All laboratory measurements were performed by routine methods or as per the user manual of the test kits in a commercial laboratory (IPE Center for Clinical Laboratory, Beijing, China) within 24 hours.

Fasting glucose was determined by the glucose oxidase-peroxidase method. The diagnosis of diabetes was made per the American Diabetes Association criteria, ${ }^{21}$ when fasting glucose $\geq 7.0 \mathrm{mmol} / \mathrm{L}$ or medical history or receiving current hypoglycemic therapy.

Dyslipidemia was defined based on the Chinese Adult Dyslipidemia Prevention Guide (2007 Edition). ${ }^{22}$ Participants were diagnosed if their serum cholesterol, triglycerides, or high-density lipoprotein cholesterol-C (HDL-C) levels were greater than $6.21 \mathrm{mmol} / \mathrm{L}, 2.26 \mathrm{mmol} / \mathrm{L}$, and less than $1.03 \mathrm{mmol} / \mathrm{L}$, respectively.

HDL-C and low-density lipoprotein cholesterol-C (LDLC) were measured by the direct assay. Total cholesterol, triglyceride, and creatinine levels were established by the standard enzymatic method with Hitachi Clinical Analyzer (Hitachi 7600; Hitachi, Tokyo, Japan). The estimated glomerular filtration rate (eGFR) was calculated according to the Modification of Diet in Renal Disease formula. ${ }^{23}$ Highsensitivity C-reactive protein (hs-CRP) was measured by a high-sensitivity nephelometric assay with a Behring Nephelometer II system (Dade Behring, Marburg, Germany).

\section{Prospective Follow-Up}

Follow-up surveys were performed to investigate the outcomes from each participant. Each participant was predetermined to be followed up for five years upon enrollment. New cases of CVD events, deaths and any other outcomes that occurred during the entire follow-up period were collected by face-to-face interview. The medical history and health insurance records of each participant were reviewed by welltrained staff. The information of coronary events, stroke events and peripheral vascular events were recorded. The death records were obtained from the participant's family, medical records, and the local Center for Disease Control and Prevention (CDC). Causes of death were codified according to the principles of the 10th version of International Classification of Diseases (ICD). In this study, CVD events were defined as a composite of coronary events (ie, fatal myocardial infarction, non-fatal myocardial infarction, percutaneous coronary intervention, coronary artery bypass surgery or sudden cardiac death), stroke events (ie, fatal or non-fatal stroke) and peripheral vascular events (ie, peripheral vascular surgery). ${ }^{24}$ Death was classified as cardiovascular or non-cardiovascular. Causes of CVD deaths included stroke, myocardial infarction and sudden cardiac death. NonCVD death included cancer, accident, infection and other non-CVD causes. All CVD events and causes of deaths were adjudicated by a panel of two physicians.

\section{Statistical Analysis}

Qualitative variables were presented as either absolute numbers or percentages and they were analyzed by the chisquared test. For qualitative data with non-normal distribution, medians (inter-quartile range, IQR) were reported and the differences of median among groups were assessed by the Kruskal-Wallis test. Quantitative data were expressed as mean \pm SD (standard deviation) and the differences among groups were assessed by ANOVA if the data followed a normal distribution.

The hazard ratios (HRs) and 95\% confidence interval (CI) of CVD event, CVD death and all-cause death were calculated according to homocysteine levels using the Cox regression model. The HR for every $5 \mu \mathrm{mol} / \mathrm{L}$ increment was also estimated using homocysteine as a continuous variable. The Cox regression model comprised the following scenarios: crude (unadjusted), model 1 (adjusted for sex and age) and model 2 (adjusted for sex, age, smoking, alcohol consumption, glucose, Hs-CRP, dyslipidemia, eGFR, pulse pressure, hypoglycemic therapy, lipidlowering therapy and antihypertensive therapy). Based on model 2, the area under the curve (AUC) of the receiveroperating characteristic (ROC) curve was calculated to evaluate the effect of the model. $p<0.05$ (two-tailed) was considered statistically significant.

\section{Results}

\section{Baseline Information and Homocysteine Level}

In total, 2468 residents were invited to participate in the baseline interview and questionnaire survey. Among them, 
1010 people were withdrawn as they refused to have their blood sampled, 37 people were excluded due to the lack of some important information, and 164 people were excluded because of a history of stroke or myocardial infarction. Eventually, 1257 residents were enrolled, and their data were carefully collected and analyzed. More females $(707,56.2 \%)$ than males $(505,43.8 \%)$ were included in this study. The average age of participants was $69.16( \pm 8.10)$ years. As shown in Table 1 , the participants with elevated homocysteine level were more likely to smoke or drink alcohol, less likely to exercise frequently, or conduct lipid lowering therapy or hypoglycemic therapy. Some other conventional risk factors, such as dyslipidemia, diabetes, hypertension, did not show significant relevance with homocysteine level. Males or elders were likely to have higher homocysteine levels than females or younger participants. More information regarding the homocysteine concentrations in different age and gender groups can be found in Table 2. Male participants had higher homocysteine level than that of females. No significant differences of homocysteine level and prevalence of hyperhomocysteinemia were found among different age groups of males. However, this was not the case in female participants. Age 55-60 group had lower level than other age groups in terms of homocysteine concentration and prevalence of hyperhomocysteinemia.

\section{Homocysteine Levels and CVD Events, and Death}

Over the 6081.8 person years and a median of $4.84( \pm 1.48)$ years' follow-up, 155 deaths occurred, and 266 adverse outcomes were identified, including 173 CVD events (62 CVD death) and 93 non-CVD deaths. The follow-up rate was $87.5 \%$, losing contact with 156 participants. Of the 173 CVD events, 58 (33.5\%) participants had coronary events, $113(65.3 \%)$ participants had stroke events and 2 $(1.2 \%)$ participants had peripheral vascular events. The detailed outcomes of the 1257 participants stratified by homocysteine levels were presented in Figure 1.

Table I Characteristics of the Participants by Different Serum Homocysteine Levels

\begin{tabular}{|c|c|c|c|c|c|}
\hline \multirow[t]{3}{*}{ Variable } & \multirow{3}{*}{ Total $(n=1257)$} & \multicolumn{3}{|c|}{ Homocysteine Level } & \multirow[t]{3}{*}{$p$-value } \\
\hline & & $<15 \mu \mathrm{mol} / \mathrm{L}$ & $<30 \mu \mathrm{mol} / \mathrm{L}$ & $\geq 30 \mu \mathrm{mol} / \mathrm{L}$ & \\
\hline & & $(n=601)$ & $(n=505)$ & $(n=|5|)$ & \\
\hline Age (years) & $69.2 \pm 8.1$ & $67.66 \pm 7.66$ & $70.47 \pm 8.22 *$ & $70.79 \pm 8.33^{*}$ & $<0.001$ \\
\hline Male & $550(43.8 \%)$ & $203(33.8 \%)$ & $253(50.1 \%)^{*}$ & $94(62.3 \%)^{*}$ & $<0.001$ \\
\hline Female & 707 (56.2\%) & $398(66.2 \%)$ & $252(49.9 \%)^{*}$ & $57(37.7 \%)^{*}$ & $<0.001$ \\
\hline Smoking & $386(30.7 \%)$ & $|4|(23.5 \%)$ & $170(33.7 \%)^{*}$ & 75 (49.7\%)* & $<0.001$ \\
\hline Drinking & $386(30.7 \%)$ & $155(25.8 \%)$ & $166(32.9 \%)^{*}$ & 65 (43.0\%)* & $<0.001$ \\
\hline Physical activity $>$ Ih/day & $572(45.5 \%)$ & $284(47.3 \%)$ & $225(44.6 \%)$ & $63(41.7 \%)$ & 0.407 \\
\hline Dyslipidemia & $736(58.6 \%)$ & $34 \mid(56.7 \%)$ & $301(59.6 \%)$ & $94(62.3 \%)$ & 0.387 \\
\hline Lipid-lowering therapy & $35(4.8 \%)$ & $24(7.0 \%)$ & $10(3.3 \%)$ & I (I.I\%)* & 0.017 \\
\hline Diabetes & $218(17.3 \%)$ & $120(20.0 \%)$ & 78 (I5.4\%) & $20(13.2 \%)$ & 0.052 \\
\hline Hypoglycemic therapy & $107(8.5 \%)$ & $71(11.8 \%)$ & $30(5.9 \%)^{*}$ & $6(4.0 \%)^{*}$ & 0.003 \\
\hline Hypertension & $762(60.6 \%)$ & 357 (59.4\%) & $314(62.2 \%)$ & 91 (60.3\%) & 0.639 \\
\hline Antihypertensive therapy & 345 (45.3\%) & $179(50.1 \%)$ & $124(39.5 \%)$ & $42(46.2 \%)$ & 0.151 \\
\hline Pulse pressure $(\mathrm{mmHg})$ & $62.2 \pm 17.2$ & $60.7 \pm 16.8$ & $63.9 \pm 17.5^{*}$ & $62.5 \pm 17.2$ & 0.009 \\
\hline Homocysteine $(\mu \mathrm{mol} / \mathrm{l})$ & $16.3(\mid 2.9-21.5)$ & $12.8(10.8-14.5)$ & $19.3(17.7-22.6)^{*}$ & $43.5(36.1-63.1)^{*}$ & $<0.001$ \\
\hline tCholesterol (mmol/L) & $5.89 \pm 1.15$ & $5.93 \pm 1.15$ & $5.83 \pm 1.15$ & $5.93 \pm 1.13$ & 0.317 \\
\hline Triglyceride $(\mathrm{mmol} / \mathrm{L})$ & $\mid .3 \mathrm{I}(0.97-\mid .88)$ & $1.33(0.98-1.89)$ & $1.30(0.96-1.91)$ & $1.25(0.95-1.8 \mathrm{I})$ & 0.889 \\
\hline $\mathrm{HDL}-\mathrm{C}(\mathrm{mmol} / \mathrm{L})$ & $1.23 \pm 0.27$ & $1.24 \pm 0.27$ & $\mathrm{I} .2 \mathrm{I} \pm 0.28$ & $1.22 \pm 0.28$ & 0.090 \\
\hline LDL-C (mmol/L) & $2.96 \pm 0.75$ & $3.01 \pm 0.75$ & $2.91 \pm 0.73$ & $2.92 \pm 0.77$ & 0.096 \\
\hline Glucose (mmol/L) & $5.72 \pm 1.66$ & $5.84 \pm 1.71$ & $5.62 \pm 1.58$ & $5.58 \pm 1.73$ & 0.057 \\
\hline hsCRP (mg/L) & $0.03(0.01-0.17)$ & $0.02(0.01-0.08)$ & $0.03(0.0 \mathrm{I}-0.2 \mathrm{I})$ & $0.04(0.01-0.16)^{*}$ & 0.004 \\
\hline eGFR & $58.80 \pm 15.37$ & $52.32 \pm 17.52$ & $56.05 \pm 12.25 *$ & $53.97 \pm 12.13^{*}$ & $<0.001$ \\
\hline BMI $\left(\mathrm{kg} / \mathrm{m}^{2}\right)$ & $24.07 \pm 3.91$ & $24.23 \pm 3.78$ & $23.96 \pm 4.07$ & $23.82 \pm 3.92$ & 0.383 \\
\hline
\end{tabular}

Notes: Data are shown as mean \pm SD, median with IQR, or absolute number (percentage). *Compared with normal homocysteine level (<I5 $\mu$ mol/L) statistically significant. Abbreviations: tCholesterol, total cholesterol; HDL-C, high-density lipoprotein cholesterol-C; LDL-C, low-density lipoprotein cholesterol-C; hsCRP, high-sensitivity C-reactive protein; eGFR, estimated glomerular filtration rate $\left(\mathrm{mL} / \mathrm{min}\right.$ per $\left.1.73 \mathrm{~m}^{2}\right)$; BMI, body mass index. 
Table 2 Homocysteine Levels in Different Gender and Age Groups

\begin{tabular}{|c|c|c|c|c|c|}
\hline & \multicolumn{3}{|c|}{ Homocysteine $(\mu \mathrm{mol} / \mathrm{L})$} & \multicolumn{2}{|c|}{ Hyperhomocysteinemia } \\
\hline & $\mathbf{n}$ & Median with IQR & $p$-value & n (\%) & $p$-value \\
\hline Gender & 1257 & 16.34 (|2.92-2I.47) & & $760(60.46)$ & \\
\hline Male & 550 & $18.00(14.55-24.75)^{*}$ & $<0.001$ & $392(71.27)^{*}$ & $<0.001$ \\
\hline Female & 707 & 15.27 (I2.02-19.34) & & $368(52.05)$ & \\
\hline Age (total) & 1257 & & & & \\
\hline $55 \sim$ & 185 & $15.14(12.18-19.40) \#$ & $<0.001$ & $94(50.81) \#$ & $<0.001$ \\
\hline $60 \sim$ & 229 & $14.96(11.55-19.58)$ & & II 4 (49.78) & \\
\hline $65 \sim$ & 225 & I5.9| (|2.0|-20.08) & & $129(57.33)$ & \\
\hline $70 \sim$ & 253 & $16.76(13.44-21.11)$ & & $158(62.45)$ & \\
\hline $75 \sim$ & 241 & $17.99(14.35-22.72)$ & & $174(72.20)$ & \\
\hline$\geq 80$ & 124 & I8.82 (|4.83-25.83) & & 91 (73.39) & \\
\hline Age (male) & 550 & & & & \\
\hline $55 \sim$ & 80 & 17.27 (13.49-22.85) & 0.381 & $53(66.25)$ & 0.338 \\
\hline $60 \sim$ & 92 & $16.60(13.81-28.75)$ & & $61(66.30)$ & \\
\hline $65 \sim$ & 90 & $16.77(14.16-20.05)$ & & $61(67.78)$ & \\
\hline $70 \sim$ & 118 & $18.09(14.76-24.00)$ & & $86(72.88)$ & \\
\hline $75 \sim$ & 109 & 18.54 (I5.22-24.94) & & 85 (77.98) & \\
\hline$\geq 80$ & 61 & $19.90(15.28-27.74)$ & & $46(75.4 I)$ & \\
\hline Age (female) & 707 & & & & \\
\hline $55 \sim$ & 105 & $13.65(10.93-16.40)^{\#}$ & $<0.001$ & $41(39.05)^{\#}$ & $<0.001$ \\
\hline $60 \sim$ & 137 & $13.76(10.88-17.97)$ & & $53(38.69)$ & \\
\hline $65 \sim$ & 135 & $15.16(11.07-18.48)$ & & $68(50.37)$ & \\
\hline $70 \sim$ & 135 & $15.57(12.24-19.78)$ & & $72(53.33)$ & \\
\hline $75 \sim$ & 132 & $17.22(|3.77-2| .62)$ & & $89(67.42)$ & \\
\hline$\geq 80$ & 63 & I8.24 (I3.84-24.74) & & 45 (7I.43) & \\
\hline
\end{tabular}

Notes: Hyperhomocysteinemia, concentration of total homocysteine $\geq 15 \mu \mathrm{mol} / \mathrm{L}$. *Compared with females; ${ }^{*}$ compared with other age groups.

Table 3 shows the crude and adjusted hazard ratios (HRs) with 95\% CI of different homocysteine levels on the risks of CVD events, CVD death and all-cause death in all participants. After adjusting for all confounding variables, the risk of CVD events and all-cause death of intermediate-to-severe hyperhomocysteinemia ( $\geq 30 \mu \mathrm{mol} /$ L) group was significantly higher than those of normal homocysteine levels $(<15 \mu \mathrm{mol} / \mathrm{L})$. The $\mathrm{HR}$ of $\mathrm{CVD}$ event and all-cause death were 1.68 (1.06-2.67) and 2.02 (1.26-3.24), respectively. Additionally, every $5 \mu \mathrm{mol} / \mathrm{L}$ increment in homocysteine concentration was shown to be associated with $4 \%$ and $5 \%$ higher risk of CVD events (HR 1.04, 95\% CI 1.01-1.07) and all-cause death (HR $1.05,95 \%$ CI 1.01-1.07), respectively. The results for CVD death were however borderline significant. The HRs and every $5 \mu \mathrm{mol} / \mathrm{L}$ increment in homocysteine concentration were 1.97 (95\% CI 0.95-4.29) and 1.04 (95\% CI 0.99-1.09) for intermediate-to-severe hyperhomocysteinemia group, respectively. No significant association was observed between normal homocysteine level and moderate hyperhomocysteinemia regarding the risk of CVD event, CVD death, all-cause death.

\section{Homocysteine Levels and Poor Outcomes in Different Gender Groups}

Table 3 demonstrates the relationship between different homocysteine levels and the risks of adverse outcomes by gender. Under Model 2 (adjusting all confounding effects), intermediate-to-severe hyperhomocysteinemia increased the risks of CVD event (HR 2.07, 95\% CI 1.01-4.26) and all-cause death (HR 3.08, 95\% CI 1.56-6.07) in males, while it was opposite for the females (HR 1.59, 95\% CI 0.83-3.04 for CVD event and HR 0.90, 95\% CI 0.52-6.07 for all-cause death). In addition, similar results were observed when homocysteine was analyzed as a continuous variable. The dose-response analysis showed that an increment of $5 \mu \mathrm{mol} / \mathrm{L}$ homocysteine could increase the risk of CVD event and the risk of all-cause 


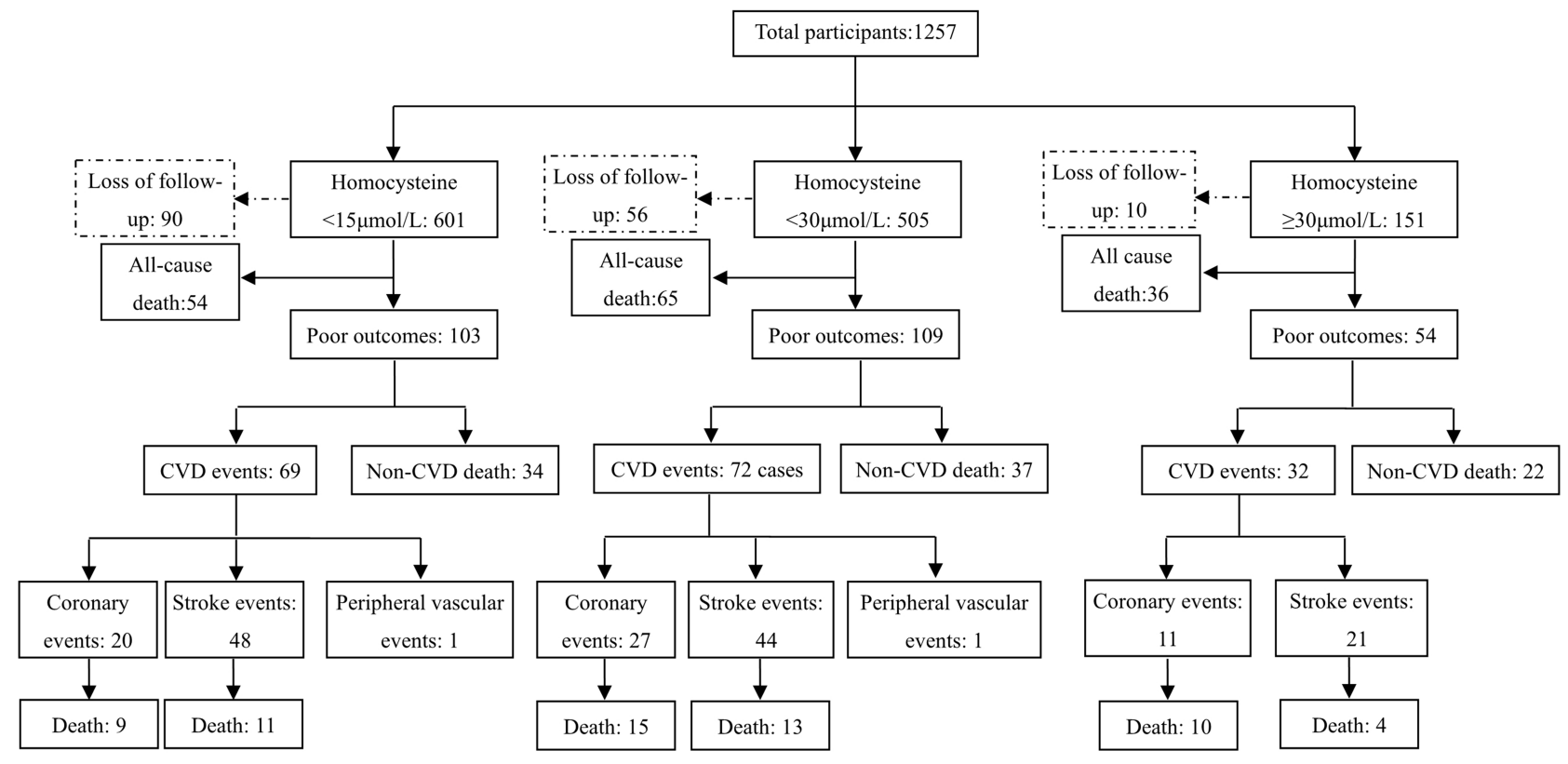

Figure I Outcome events of 1257 participants.

death in males by $5 \%$ (HR 1.05, 95\% CI 1.01-1.08) and $6 \%$ (HR 1.06, 95\% CI 1.02-1.09), respectively.

\section{ROC Analysis}

To obtain the optimal cutoff point and predict the risk of CVD events, receiver operating characteristic (ROC) curves were constructed (Figure 2). In the fully adjusted Model 2, the areas under the curve (AUC) of the ROC curve were shown in Table 4. The optimal cutoff points with highest Youden's index (specificity + sensitivity-1) were $13.20 \mu \mathrm{mol} /$ $\mathrm{L}, 15.08 \mu \mathrm{mol} / \mathrm{L}$, and $12.91 \mu \mathrm{mol} / \mathrm{L}$ for CVD events, stroke, and coronary heart disease, respectively.

\section{Discussion}

In this study, strong associations were observed between homocysteine level and CVD events or all-cause mortality in elder Chinese without history of ischemia or congestive heart failure (CHF). This relationship persisted after adjusting for some conventional risk factors. People with a high homocysteine level had a higher total and cardiovascular mortality. Our data provided solid evidence that elevated homocysteine level was an independent risk factor of all-cause mortality in Chinese residents.

This study was an extension of BLSA initiated in 1992, which aimed to define hyperhomocysteinemia for the use of homocysteine screening targeting more effective prediction and primary prevention. Considering no general agreement on the optimal value of homocysteine that confers pertinent outcomes, refer to the statement by the American Heart Association, ${ }^{13}$ a serum homocysteine level of $15 \mu \mathrm{mol} / \mathrm{L}$ was predetermined as a cutoff point for screening. Serum homocysteine less than $15 \mu \mathrm{mol} / \mathrm{L}$ should be corresponded to the 90th percentile of the distribution of normal homocysteine level.

Previous studies have shown that homocysteine level was a moderately sensitive and specific index to predict the prognosis with an optimal cutoff value at 19.95 $\mu \mathrm{mol} / \mathrm{L}$ with sensitivity of $58.2 \%$ and specificity of $80.3 \%{ }^{25}$ Another study suggested the homocysteine concentration $11.11 \mu \mathrm{mol} / \mathrm{L}$ as the optimal cutoff point due to better specificity and sensitivity for predicting allcause death. ${ }^{26}$ In this study, after adjusting for sex, age, smoking, alcohol drinking, glucose, hs-CRP, dyslipidemia, eGFR, pulse pressure, hypoglycemic therapy, lipidlowering therapy, and antihypertensive therapy, the fully adjusted model suggested the optimal cutoff points with the highest Youden's index were $13.20 \mu \mathrm{mol} / \mathrm{L}$, $15.08 \mu \mathrm{mol} / \mathrm{L}$, and $12.91 \mu \mathrm{mol} / \mathrm{L}$ for CVD events, stroke, and coronary heart disease, respectively. These optimal cutoff points were close to the predetermined cutoff point values of this study. Hence, we think that the predetermined cutoff point is reasonable.

Generally, the optimal cutoff point of homocysteine level should be determined by the maximum value of Youden's index. However, this data-oriented selection of optimal cutoff point is prone to biases caused by study population, 
Table 3 The Relationship Between Homocysteine Level and CVD Events and Death

\begin{tabular}{|c|c|c|c|c|c|}
\hline & \multicolumn{4}{|l|}{ Homocysteine Level } & \multirow[t]{2}{*}{$\mathrm{HR} / 5 \mu \mathrm{mol} / \mathrm{L} \mathrm{tHcy}$} \\
\hline & $<15 \mu \mathrm{mol} / \mathrm{L}(\mathrm{n}=60 \mathrm{I})$ & $<30 \mu \mathrm{mol} / \mathrm{L}(\mathrm{n}=505)$ & $\geq 30 \mu \mathrm{mol} / \mathrm{L}(\mathrm{n}=15 \mathrm{I})$ & $p$ trend* & \\
\hline \multicolumn{6}{|c|}{ CVD event (total) } \\
\hline Case & 67 & 69 & 32 & & \\
\hline Person-year & 3210.4 & 2549.9 & 778.4 & & \\
\hline Crude & 1.00 & $1.34(0.96-1.87)$ & $2.18(1.43-3.32)$ & $<0.001$ & $1.06(1.03-1.09)$ \\
\hline Model I & 1.00 & I.18 (0.84-I.67) & $1.93(1.25-2.96)$ & 0.006 & $1.05(1.02-1.08)$ \\
\hline Model 2 & 1.00 & $1.12(0.78-1.61)$ & $1.68(1.06-2.67)$ & 0.044 & $1.04(1.01-1.07)$ \\
\hline \multicolumn{6}{|c|}{ CVD event (male) } \\
\hline Case & 20 & 38 & 19 & & \\
\hline Person-year & 1025.3 & 1172.4 & 415.0 & & \\
\hline Crude & 1.00 & $1.69(1.01-2.90)$ & $2.42(1.29-4.54)$ & 0.005 & $1.04(1.01-1.08)$ \\
\hline Model I & 1.00 & $1.62(0.94-2.79)$ & $2.38(2.27-4.45)$ & 0.006 & $1.05(1.27-4.45)$ \\
\hline Model 2 & 1.00 & $1.65(0.91-3.00)$ & $2.07(1.01-4.26)$ & 0.046 & $1.05(1.01-1.08)$ \\
\hline \multicolumn{6}{|c|}{ CVD event (female) } \\
\hline Case & 47 & 31 & 13 & & \\
\hline Person-year & 2012.2 & 1189.4 & 267.1 & & \\
\hline Crude & 1.00 & $1.13(0.72-1.77)$ & $2.12(1.14-3.91)$ & 0.047 & $1.02(0.96-1.08)$ \\
\hline Model I & 1.00 & $0.93(0.58-1.47)$ & $1.62(0.87-3.04)$ & 0.334 & $1.00(0.93-1.07)$ \\
\hline Model 2 & 1.00 & $0.92(0.56-1.49)$ & $1.59(0.83-3.04)$ & 0.365 & $0.99(0.92-1.06)$ \\
\hline \multicolumn{6}{|c|}{ CVD death (total) } \\
\hline Case & 20 & 28 & 14 & & \\
\hline Person-year & 3150.6 & 2467.4 & 734.8 & & \\
\hline Crude & 1.00 & $1.81(1.01-3.20)$ & $3.04(1.54-6.02)$ & 0.001 & $1.06(1.01-1.10)$ \\
\hline Model I & 1.00 & $1.30(0.73-2.34)$ & $2.21(1.10-4.42)$ & 0.033 & $1.05(1.00-1.40)$ \\
\hline Model 2 & 1.00 & $\mathrm{I} .4 \mathrm{I}(0.75-2.66)$ & $1.97(0.95-4.29)$ & 0.065 & $1.04(0.99-1.09)$ \\
\hline \multicolumn{6}{|c|}{ CVD death (male) } \\
\hline Case & 6 & 15 & 9 & & \\
\hline Person-year & 1061.8 & 1222.2 & 443.5 & & \\
\hline Crude & 1.00 & $2.19(0.85-5.66)$ & $3.64(1.30-10.23)$ & 0.012 & $1.06(1.01-1.11)$ \\
\hline Model I & 1.00 & $1.90(0.74-4.91)$ & $3.51(1.25-9.86)$ & 0.016 & $1.06(1.01-1.12)$ \\
\hline Model 2 & 1.00 & $2.11(0.69-6.42)$ & $2.83(0.78-10.21)$ & 0.117 & $1.05(0.99-1.12)$ \\
\hline \multicolumn{6}{|c|}{ CVD death (female) } \\
\hline Case & 14 & 13 & 5 & & \\
\hline Person-year & 2088.8 & 1245.2 & 291.3 & & \\
\hline Crude & 1.00 & $1.57(0.74-3.34)$ & $2.57(0.93-7.13)$ & 0.059 & $1.03(0.93-1.13)$ \\
\hline Model I & 1.00 & $0.99(0.46-2.13)$ & $1.34(0.47-3.84)$ & 0.681 & $0.98(0.87-1.12)$ \\
\hline Model 2 & 1.00 & $1.09(0.46-2.58)$ & $1.29(0.4 I-4.09)$ & 0.671 & $0.98(0.87-1.1 \mathrm{I})$ \\
\hline \multicolumn{6}{|c|}{ All-cause death (total) } \\
\hline Case & 54 & 65 & 36 & & \\
\hline Person-year & 3150.6 & 2467.4 & 734.8 & & \\
\hline Crude & 1.00 & $1.56(1.09-2.23)$ & $2.91(1.91-4.44)$ & $<0.001$ & $1.07(1.03-1.09)$ \\
\hline Model I & 1.00 & $1.10(0.76-1.59)$ & $2.08(1.36-3.19)$ & 0.003 & $1.06(1.02-1.08)$ \\
\hline Model 2 & 1.00 & $1.17(0.79-1.73)$ & $2.02(1.26-3.24)$ & 0.007 & $1.05(1.01-1.07)$ \\
\hline \multicolumn{6}{|c|}{ All-cause death (male) } \\
\hline Case & 20 & 36 & 26 & & \\
\hline Person-year & 1061.8 & 1222.2 & 443.5 & & \\
\hline Crude & 1.00 & $1.59(0.92-2.75)$ & $3.20(1.78-5.72)$ & $<0.001$ & $1.07(1.03-1.09)$ \\
\hline
\end{tabular}

(Continued) 
Table 3 (Continued).

\begin{tabular}{|c|c|c|c|c|c|}
\hline & \multicolumn{4}{|l|}{ Homocysteine Level } & \multirow[t]{2}{*}{$\mathrm{HR} / 5 \mu \mathrm{mol} / \mathrm{L}$ tHcy } \\
\hline & $<15 \mu \mathrm{mol} / \mathrm{L}(\mathrm{n}=60 \mathrm{I})$ & $<30 \mu \mathrm{mol} / \mathrm{L}(\mathrm{n}=505)$ & $\geq 30 \mu \mathrm{mol} / \mathrm{L}(\mathrm{n}=15 \mathrm{I})$ & $p$ trend* & \\
\hline Model I & 1.00 & $1.38(0.80-2.39)$ & $3.11(1.73-5.57)$ & $<0.001$ & $1.06(1.03-1.09)$ \\
\hline Model 2 & 1.00 & $1.47(0.80-2.68)$ & $3.08(1.56-6.07)$ & 0.001 & $1.06(1.02-1.09)$ \\
\hline \multicolumn{6}{|c|}{ All-cause death (female) } \\
\hline Case & 34 & 29 & 10 & & \\
\hline Person-year & 2088.8 & 1245.2 & 291.3 & & \\
\hline Crude & 1.00 & I.44 (0.88-2.37) & $2.12(1.05-4.28)$ & 0.025 & $1.03(0.97-1.10)$ \\
\hline Model I & 1.00 & $0.87(0.53-1.47)$ & $1.07(0.52-2.22)$ & 0.954 & $0.98(0.90-1.07)$ \\
\hline Model 2 & 1.00 & $0.90(0.4 \mathrm{I}-\mathrm{I} .98)$ & $0.90(0.52-1.58)$ & $0.74 I$ & $0.97(0.89-1.06)$ \\
\hline
\end{tabular}

Notes: *Estimated using the category numbers (I, 2 and 3) as a continuous variable; CVD: cardiovascular disease; Crude: unadjusted; Model I: adjusted for sex and age; Model 2: adjusted for sex, age, smoking, alcohol drinking, glucose, hsCRP, dyslipidemia, eGFR, pulse pressure, hypoglycemic therapy, lipid-lowering therapy and antihypertensive therapy.

sample size, the accuracy of measurements, etc. Chances are system errors may lead to either overestimation or underestimation of the sensitivity and specificity. Hence, it is necessary to use caution when the results are applied for clinical prediction and primary prevention.

During the 6081.8 person years follow-up for 1257 participants with a median of $4.84 \pm 1.48$ years, 173 CVD events were identified. $12.3 \%$ of the participants died and $40.0 \%$ of these deaths were due to CVD. Participants with intermediate-to-severe homocysteine level were at higher risk of death from all cause-death and CVD death than normal homocysteine group. Although it is still debatable whether homocysteine is a causal factor or a marker of CVD and mortality, evidence from previous studies had confirmed our findings that serum total homocysteine concentrations are predictive of CVD and/or mortality. ${ }^{12,27}$ All-cause mortality was also proven to be strongly associated with the level of plasma homocysteine. The cutoff point of homocysteine concentration and HR were determined to be $11.84 \mathrm{mmol} / \mathrm{L}$ and 2.4 (95\% CI,1.76-3.32), respectively. ${ }^{13}$

The difference between reported values may be statistically significant. However, when these values were compared, one should use caution and carefully study the characteristics of participants, which may lead to different cutoff point and HR values. Given the features of the study participants, such as more severe chronic kidney disease, older age, or vitamin $B_{12}$ deficiency, the HR of those participants would be greater. ${ }^{27,28}$ In the present study, the participants consisted of regular residents living in Beijing, aged 55 or above and without history of ischemia or congestive heart failure (CHF). They could be healthier than those patients with CHF. Hence, the HR value of the regular residents was lower than that of the patients with $\mathrm{CHF}^{29}$

Other than age and gender, in this study, we tested hypertension, diabetes, smoking, drinking and other conventional risk factors as possible confounding factors of hyperhomocysteinemia. We found that elevated homocysteine level increased the risks of CVD events and all-cause death in males, but not in females. In addition, males or elders were likely to have higher homocysteine levels than females or younger participants. This finding was in accordance with a prospective study, which showed that intermediate-to-severe hyperhomocysteinemia increased the risk of ischemic stroke in the males (HR: $6.11,95 \%$ CI 3.44-10.85) but not in the females (HR: $1.84,95 \%$ CI 0.52-6.64). ${ }^{8}$ A supportive mechanism study revealed that hyperhomocysteinemia was associated with carotid resistive index, a surrogate marker of cerebral vascular resistance, especially in elderly men. ${ }^{30}$ On the contrary, the Amsterdam study showed that elevated homocysteine was associated with an increased risk of all-cause death in females (HR: 2.97, 95\% CI 1.36-6.49), but not in males (HR: $0.66,95 \%$ CI $0.22-1.98) .{ }^{31}$ Therefore, we suggest that age and gender may be effecting modifiers and the adverse outcomes of hyperhomocysteine on males or females need further study. It is well known that homocysteine is an intermediate metabolite of methionine with three metabolic pathways. ${ }^{32}$ They are 1) catalyzed by cystathionine beta synthetase to cysteine, 2) methylated by betaine homocysteine methyltransferase to methionine, and 3) catalyzed by methionine synthase into methionine. Thus, the homocysteine level varies depending on age, diet 

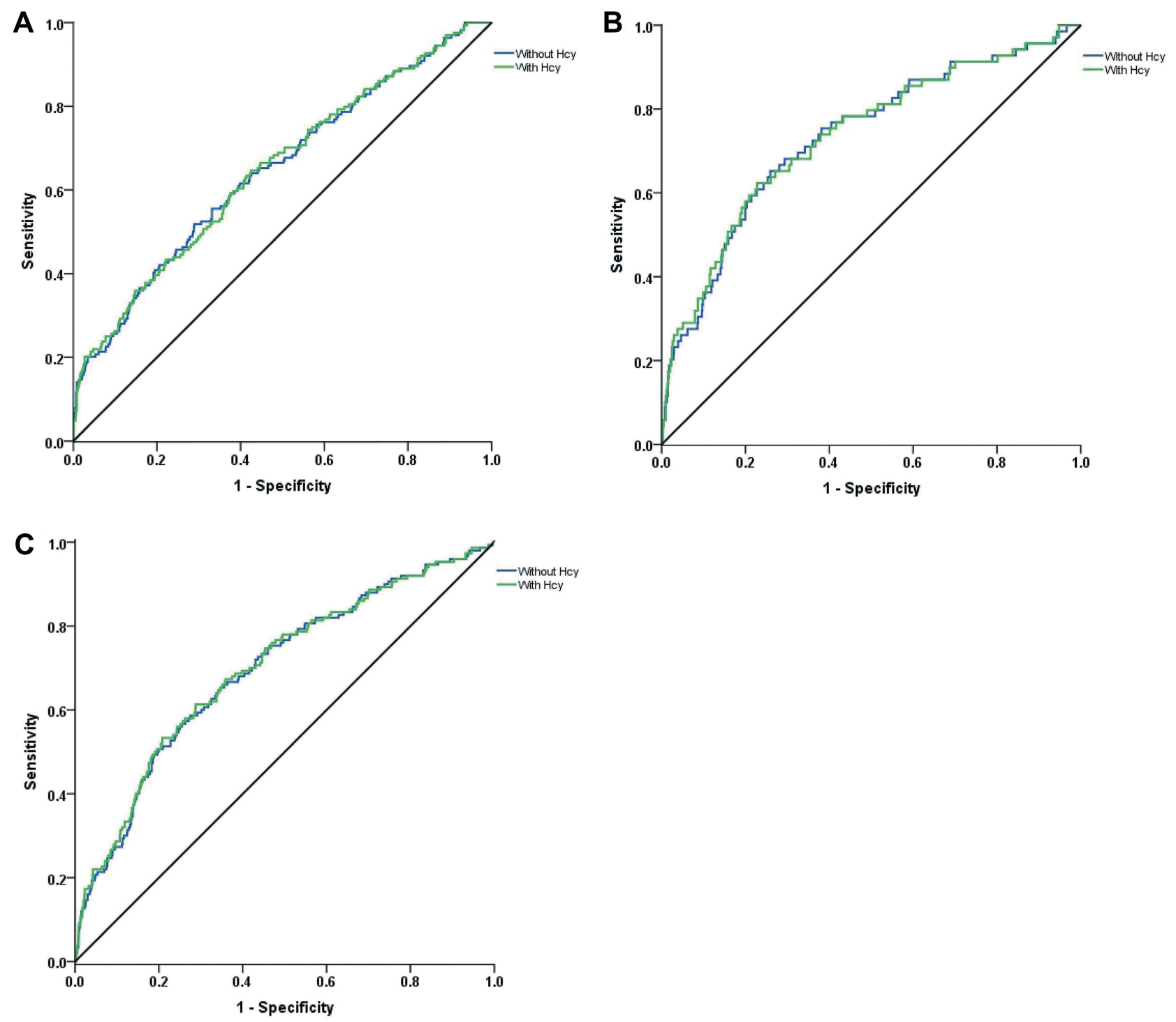

Figure 2 Receiver-operating characteristic curves for (A) CVD events, (B) CVD death, and (C) all-cause death during 4.84-year follow-up. For each endpoint, curves are based on prediction models adjusted for conventional risk factors (sex, age, smoking, alcohol drinking, glucose, hs-CRP, dyslipidemia, eGFR, pulse pressure, hypoglycemic therapy, lipid-lowering therapy and antihypertensive therapy) with or without homocysteine (Hcy).

(eg, folic acid deficiency), and genetic background (eg, gene mutation of enzymes of homocysteine metabolic pathways). ${ }^{33}$ Hence, in future studies, a better understanding of human's metabolism of homocysteine as well as the

Table 4 The Area Under the Curve of ROC Curve

\begin{tabular}{|l|l|l|}
\hline & $\begin{array}{l}\text { Conventional Risk Factors } \\
\text { Without Hcy }\end{array}$ & $\begin{array}{l}\text { Conventional Risk } \\
\text { Factors With Hcy }\end{array}$ \\
\hline Figure 2-A & $0.632(0.559-0.695)$ & $0.649(0.610-0.696)$ \\
Figure 2-B & $0.733(0.668-0.805)$ & $0.739(0.669-0.808)$ \\
Figure 2-C & $0.684(0.648-0.742)$ & $0.699(0.652-0.746)$ \\
\hline
\end{tabular}

Notes: Data were shown as estimate $(95 \% \mathrm{Cl})$.

Abbreviation: Hcy: homocysteine pathogenic pathways in protein, DNA, and RNA level should lead to new strategies for more targeted prevention and gene therapy.

The limitations of this study revolve around the following aspects. Although we have followed 1257 participants for a total of 6081.8 person-years, due to the withdrawal of some participants at baseline investigation, the sample size of this study was smaller than the original research design. Chances are the representativeness of the sample could be weaker and the potential bias of the results may be greater than they were expected. Serum homocysteine level may be influenced by age, diet, genetic background, and/or the use of medications. A single test of serum homocysteine 
cannot provide enough information for us to establish causal relationship between homocysteine and CVD. In addition, the participants were limited to the residents in Beijing. Hence, the results of this study should be extrapolated cautiously to other population or ethnic groups.

\section{Conclusion}

Intermediate-to-severe hyperhomocysteinemia was significantly associated with CVD events and all-cause death in elderly Chinese population free of CVD history. The positive association was pronounced among males.

\section{Abbreviations}

CVD, cardiovascular diseases; CHF, congestive heart failure; HR, hazard ratio; CI, confidence interval; IQR, interquartile range; hs-CRP, high-sensitivity $\mathrm{C}$-reactive protein.

\section{Funding}

This study was supported by grants from the Commission of Science and Technology of Beijing (Grant No: D121100004912002), Beijing Natural Science Foundation (Grant No: 7152068) and the project for collaboration between basis and clinic of Capital Medical University (Grant No: 17JL69).

\section{Disclosure}

The authors report no conflicts of interest in this work.

\section{References}

1. Roth GA, Huffman MD, Moran AE, et al. Global and regional patterns in cardiovascular mortality from 1990 to 2013. Circulation. 2015;132 (17):1667-1678. doi:10.1161/CIRCULATIONAHA.114.008720

2. Sun H, Zou X, Liu L. Epidemiological factors of stroke: a survey of the current status in china. J Stroke. 2013;15(2):109-114. doi:10.5853/ jos.2013.15.2.109

3. Lloyd-Jones DM. Cardiovascular risk prediction: basic concepts, current status, and future directions. Circulation. 2010;121 (15):1768-1777. doi:10.1161/CIRCULATIONAHA.109.849166

4. Peng YP, Huang MY, Xue YJ, Pan JL, Lin C. Association of hyperhomocysteinemia with increased coronary microcirculatory resistance and poor short-term prognosis of patients with acute myocardial infarction after elective percutaneous coronary intervention. Biomed Res Int. 2020;2020:1710452. doi:10.1155/2020/1710452

5. Agoston-Coldea L, Mocan T, Gatfosse M, Lupu S, Dumitrascu DL. Plasma homocysteine and the severity of heart failure in patients with previous myocardial infarction. Cardiol J. 2011;18(1):55-62.

6. He Y, Li Y, Chen Y, Feng L, Nie Z. Homocysteine level and risk of different stroke types: a meta-analysis of prospective observational studies. Nutr Metab Cardiovasc Dis. 2014;24(11):1158-1165. doi:10.1016/j.numecd.2014.05.011

7. Zhang W, Sun K, Chen J, et al. High plasma homocysteine levels contribute to the risk of stroke recurrence and all-cause mortality in a large prospective stroke population. Clin sci. 2009;118(3):187-194. doi:10.1042/CS20090142
8. Han L, Wu Q, Wang C, et al. Homocysteine, ischemic stroke, and coronary heart disease in hypertensive patients: a population-based, prospective cohort study. Stroke. 2015;46(7):1777-1786. doi:10.1161/STROKEAHA.115.009111

9. Wang CY, Chen ZW, Zhang T, et al. Elevated plasma homocysteine level is associated with ischemic stroke in Chinese hypertensive patients. Eur J Intern Med. 2014;25(6):538-544. doi:10.1016/j. ejim.2014.04.011

10. Marti-Carvajal AJ, Sola I, Lathyris D. Homocysteine-lowering interventions for preventing cardiovascular events. Cochrane Database Syst Rev. 2015;1:Cd006612.

11. Huo Y, Li J, Qin X, et al. Efficacy of folic acid therapy in primary prevention of stroke among adults with hypertension in China: the CSPPT randomized clinical trial. JAMA. 2015;313(13):1325-1335. doi:10.1001/jama.2015.2274

12. Shi Z, Guan Y, Huo YR, et al. Elevated total homocysteine levels in acute ischemic stroke are associated with long-term mortality. Stroke. 2015;46(9):2419-2425. doi:10.1161/STROKEAHA.115.009136

13. Sun Y, Chien KL, Hsu HC, Su TC, Chen MF, Lee YT. Use of serum homocysteine to predict stroke, coronary heart disease and death in ethnic Chinese. 12-year prospective cohort study. Circ j. 2009;73 (8):1423-1430. doi:10.1253/circj.CJ-08-1077

14. Towfighi A, Arshi B, Markovic D, Ovbiagele B. Homocysteinelowering therapy and risk of recurrent stroke, myocardial infarction and death: the impact of age in the VISP trial. Cerebrovascular Dis. 2014;37(4):263-267. doi:10.1159/000360153

15. Tang Z, Zhou T, Luo Y, et al. Risk factors for cerebrovascular disease mortality among the elderly in Beijing: a competing risk analysis. PLoS One. 2014;9(2):e87884. doi:10.1371/journal.pone.0087884

16. Zhang Z, Fang X, Hua Y, et al. Combined effect of hyperhomocysteinemia and hypertension on the presence of early carotid artery atherosclerosis. J Stroke Cerebrovascular Dis. 2016;25 (5):1254-1262. doi:10.1016/j.jstrokecerebrovasdis.2016.01.037

17. Zimmer Z, Fang X, Tang Z. Fifteen-year disability trends among older persons in the Beijing municipality of China. J Aging Health. 2014;26(2):207-230. doi:10.1177/0898264313513609

18. Endris T, Worede A, Asmelash D. Prevalence of diabetes mellitus, prediabetes and its associated factors in dessie town, northeast ethiopia: a community-based study. Diabetes Metab Syndrome Obesity. 2019;12:2799-2809. doi:10.2147/DMSO.S225854

19. Lai MMY, Sharman MJ, Ames DJ, et al. Relationship of established cardiovascular risk factors and peripheral biomarkers on cognitive function in adults at risk of cognitive deterioration. $J$ Alzheimers Dis. 2020;74(1):163-171. doi:10.3233/JAD-190953

20. Yu S, Chen $\mathrm{Y}$, Yang $\mathrm{H}$, Guo $\mathrm{X}$, Zheng L, Sun $\mathrm{Y}$. Hyperhomocysteinemia accompany with metabolic syndrome increase the risk of left ventricular hypertrophy in rural Chinese. BMC Cardiovasc Disord. 2020;20(1):44. doi:10.1186/s12872-02001350-2

21. Ameriacan Diabetes Association. Standards of medical care in diabetes-2011. Diabetes Care. 2011;34(Suppl 1):S11-S61. doi:10.2337/ dc11-S011

22. Joint Committee. [Chinese guidelines on prevention and treatment of dyslipidemia in adults]. Zhonghua Xin Xue Guan Bing Za Zhi. 2007;35(5):390-419. Chinese.

23. Tiongco RE, Bituin A, Arceo E, Rivera N, Singian E. Salivary glucose as a non-invasive biomarker of type 2 diabetes mellitus. $J$ clin exp dent. 2018;10(9):e902-e907. doi:10.4317/jced.55009

24. Polak JF, Pencina MJ, Pencina KM, O'Donnell CJ, Wolf PA, D'Agostino RB. Carotid-wall intima-media thickness and cardiovascular events. $N$ Engl J Med. 2011;365(3):213-221. doi:10.1056/ NEJMoa1012592

25. Yao ES, Tang Y, Xie MJ, Wang MH, Wang H, Luo X. Elevated homocysteine level related to poor outcome after thrombolysis in acute ischemic stroke. Med Sci Monit. 2016;22:3268-3273. doi:10.12659/MSM.900010 
26. Luo JL, Chien KL, Hsu HC, et al. Association between plasma homocysteine concentration and the risk of all-cause death in adults with diastolic dysfunction in a community: a 13-year cohort study. Medicine. 2017;96(17):e6716. doi:10.1097/MD.0000000000006716

27. Wong YY, Almeida OP, McCaul KA, Yeap BB, Hankey GJ, Flicker L. Homocysteine, frailty, and all-cause mortality in older men: the health in men study. $j$ Gerontology Ser a Biol Sci Med Sci. 2013;68(5):590-598. doi:10.1093/gerona/gls211

28. Mendonca N, Jagger C, Granic A, et al. Elevated total homocysteine in all participants and plasma vitamin b12 concentrations in women are associated with all-cause and cardiovascular mortality in the very old: the newcastle 85+ study. J Gerontol a Biol Sci Med Sci. 2018;73 (9):1258-1264. doi:10.1093/gerona/gly035

29. Naruszewicz M, Jankowska EA, Zymlinski R, et al Hyperhomocysteinemia in patients with symptomatic chronic heart failure: prevalence and prognostic importance-pilot study. Atherosclerosis. 2007;194(2):408-414. doi:10.1016/j.atherosclerosis.2006.08.014
30. Okura T, Miyoshi K, Irita J, et al. Hyperhomocysteinemia is one of the risk factors associated with cerebrovascular stiffness in hypertensive patients, especially elderly males. Sci Rep. 2014;4(1):5663. doi:10.1038/srep05663

31. Swart KM, van Schoor NM, Blom HJ, Smulders YM, Lips P. Homocysteine and the risk of nursing home admission and mortality in older persons. Eur J Clin Nutr. 2012;66(2):188-195. doi:10.1038/ ejcn.2011.186

32. Loscalzo J, Handy DE. Epigenetic modifications: basic mechanisms and role in cardiovascular disease (2013 Grover Conference series). Pulm Circ. 2014;4(2):169-174. doi:10.1086/675979

33. Ganguly P, Alam SF. Role of homocysteine in the development of cardiovascular disease. Nutr J. 2015;14(1):6. doi:10.1186/1475-289114-6

\section{Publish your work in this journal}

Therapeutics and Clinical Risk Management is an international, peerreviewed journal of clinical therapeutics and risk management, focusing on concise rapid reporting of clinical studies in all therapeutic areas, outcomes, safety, and programs for the effective, safe, and sustained use of medicines. This journal is indexed on PubMed Central, CAS,
EMBase, Scopus and the Elsevier Bibliographic databases. The manuscript management system is completely online and includes a very quick and fair peer-review system, which is all easy to use. Visit http://www.dovepress.com/testimonials.php to read real quotes from published authors. 